\title{
The efficacy of adjuvant immunochemotherapy with OK-432 after curative resection of gastric cancer: an individual patient data meta-analysis of randomized controlled trials
}

\author{
Mari S. Oba $^{1,7} \cdot$ Satoshi Teramukai $^{2} \cdot$ Yasuo Ohashi $^{3} \cdot$ Kenji Ogawa $^{4} \cdot$ \\ Yoshihiko Maehara ${ }^{5} \cdot$ Junichi Sakamoto $^{6}$
}

Received: 20 October 2014/Accepted: 13 March 2015/Published online: 25 March 2015

(C) The International Gastric Cancer Association and The Japanese Gastric Cancer Association 2015

\begin{abstract}
Background OK-432 has been used as a cancer treatment for 40 years, and the immunostimulatory effects of OK432 therapy have been intensely investigated in Japan. Recently, it has received attention as a possible booster for cancer vaccine treatments. Our previous meta-analysis based on summary measures revealed a significant improvement in the survival of patients with curatively resected gastric cancer. However, it is impossible to exclude the possibility of bias due to several prognostic factors.

Methods We collected individual data for patients with stage III or stage IV gastric cancer after curative resection from 14 trials that were identified in a previous metaanalysis. Immunochemotherapy with OK-432 was compared with treatment with standard chemotherapy on an intention-to-treat basis. The primary end point was overall survival. Stratified survival analyses were performed with the trial as the stratification factor. Subgroup analyses were
\end{abstract}

Mari S. Oba

mari@yokohama-cu.ac.jp

1 Department of Biostatistics and Epidemiology, Yokohama City University, Yokohama, Japan

2 Department of Biostatistics, Graduate School of Medical Science, Kyoto Prefectural University of Medicine, Kyoto, Japan

3 Department of Integrated Science and Engineering for Sustainable Society, Chuo University, Tokyo, Japan

4 Department of Surgery, Tokyo Women's Medical University Medical Center East, Tokyo, Japan

5 Department of Surgery and Science, Graduate School of Medical Sciences, Kyushu University, Fukuoka, Japan

6 Tokai Central Hospital, Kakamigahara, Japan

7 4-57 Urafune-cho, Minami-ku, Yokohama 232-0024, Japan also performed according to the potential prognostic factors, which included pathological factors, splenectomy, and delayed-type hypersensitivity.

Results There were 796 and 726 patients in the OK-432 and control groups, respectively. The median overall survival was 42.6 months for the OK-432 group and 32.3 months for the control group. The overall hazard ratio was 0.88 (95\% confidence interval $0.77-1.00, p=0.050$ ). No factor showed a statistically significant interaction in the subgroup analyses.

Conclusions The results suggest that immunochemotherapy treatment with OK-432 could have a borderline significant effect for patients with stage III or stage IV gastric cancer after curative resection.

Keywords Adjuvant immunochemotherapy - Gastric cancer $\cdot$ OK-432 - Individual patient data $\cdot$ Meta-analysis

\section{Introduction}

OK-432 is a lyophilized preparation produced by incubating a culture of the low-virulence Su strain of group A Streptococcus pyogenes of human origin that is treated with penicillin $\mathrm{G}$ potassium. It was approved in 1975 for clinical use in cancer patients by the Japanese Ministry of Health and Welfare [1], and was indicated for (1) prolongation of survival time in patients with gastric cancer or primary lung cancer in combination with chemotherapy [2, $3]$, (2) reduction of cancerous pleural effusions or ascites in patients with gastrointestinal or lung cancer [4, 5], (3) treatment of head and neck cancer and thyroid cancer resistant to other chemotherapies [6,7], and (4) lymphangioma [8]. The antitumor activities of OK-432, in particular the immunostimulatory effects, have been 
intensely investigated in Japan, but to a much lesser extent in the USA and Europe.

The results from several clinical studies that were conducted primarily in Japan were published between 1990 and 2000. Although favorable effects after treatment were reported in anecdotal studies, no individual randomized clinical trial demonstrated a significant improvement in survival. In 2002, we investigated the efficacy of adjuvant immunochemotherapy with OK-432 in 1522 patients with curatively resected gastric cancer, and demonstrated that standard chemotherapy combined with OK-432 treatment was superior to chemotherapy alone. The 3 -year overall survival (OS) odds ratio was 0.81 (95\% confidence interval, CI, 0.65-0.99) [9]. Although the results demonstrated the benefits of combined treatment, the possibility of bias due to several prognostic factors could not be excluded since the study was performed on the basis of the tabulated data from a meta-analysis of randomized trials.

New aspects of OK-432 treatment have been investigated since the beginning of the twenty-first century, and multiple lines of evidence supporting the effects of OK-432 have been reported, including that (1) OK-432 induces dendritic cell maturation and induces cytotoxic $\mathrm{T}$ lymphocytes specific to tumors [10-12], (2) OK-432 is effective as an adjuvant to peptide vaccines such as NY-ESO-1 [13-17], and (3) OK-432 is effective as an adjuvant to cisplatin and hyperthermotherapy [18, 19]. In addition to the efficacy of adjuvant OK-432, it was also expected to overcome the suppression of regulatory $\mathrm{T}$ cells $[20,21]$, and to induce a helper $\mathrm{T}$ cell response [22]. With the increased attention on OK-432 therapy, a detailed reevaluation of the results of cancer therapy using OK-432 in previous clinical trials was determined to be important.
In this study, we collected data for individual patients who were enrolled in eligible randomized trials and reexamined the precise effects of immunotherapy using OK432 in an adjuvant setting for locally advanced disease. The current standard of treatment for patients with stage I gastric cancer after curative resection does not necessarily involve adjuvant chemotherapy, and given the results of ACTS-GC [23], chemotherapy without immunotherapy is generally sufficient for stage II gastric cancer; however, more powerful therapy is required to treat stage III gastric cancer and curatively resected stage IV gastric cancer. In this regard, we focused on patients with stage III or stage IV curatively resected gastric cancer. This reanalysis was important in order to clarify the immunological effects of OK-432, which has become widely used as a new immunotherapy and vaccine therapy for various cancers.

\section{Methods}

We performed a literature search with the MEDLINE database, presentations at meeting and inquiry for the pharmaceutical industry and research groups in August 1999. Trials were eligible if (1) patients were randomized, (2) a curative resection was scheduled, (3) standard chemotherapy was performed after tumor resection, and (4) OK-432 was administered intramuscularly or intradermally. The PRISMA diagram in Fig. 1 shows this process, and additional details regarding the trials included have been published elsewhere [9].

The corresponding authors from each trial provided the individual patient data (IPD), including sex, age, pathological tumor-node-metastasis (TNM) stage, clinical stage,
Fig. 1 Flowchart of the study design and trial selection

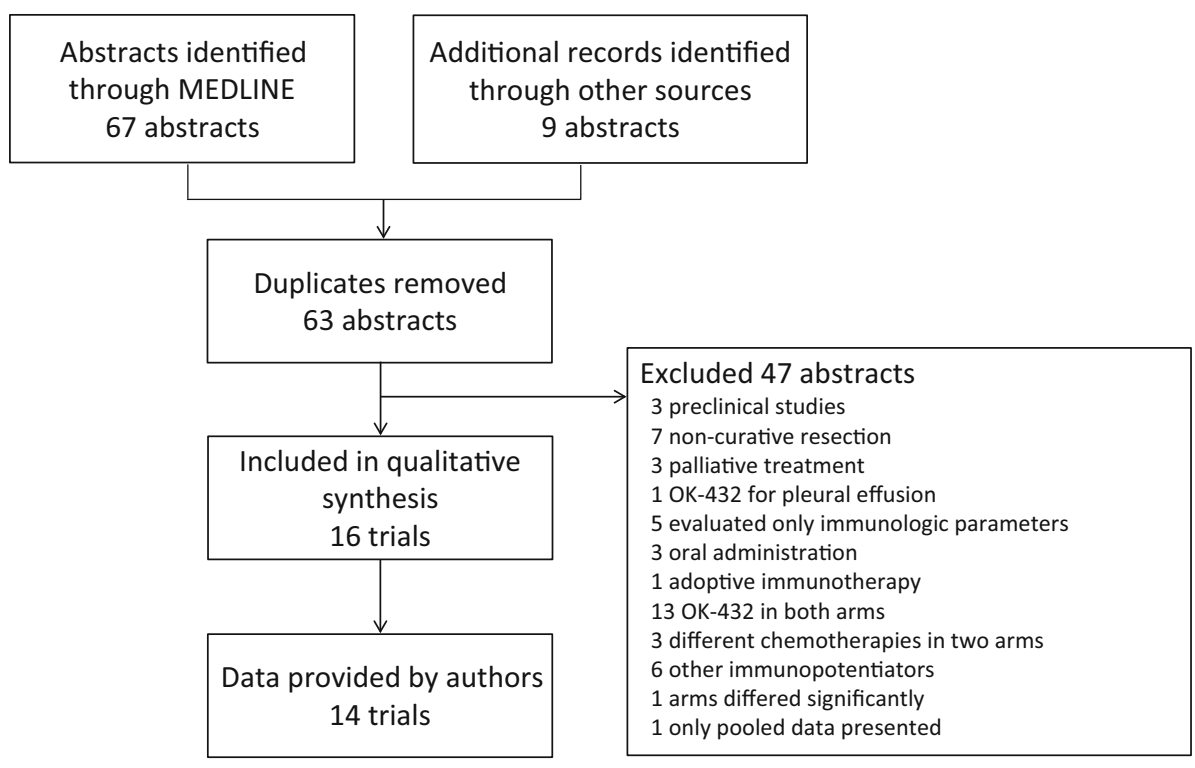


operation type, splenectomy status, delayed-type hypersensitivity reaction, date of operation, date of last followup, and survival status. The stage classification was performed according to the Japanese Classification of Gastric Carcinoma (13th edition). In this edition, stage IV cancer included a pathological T4 cancer without any metastasis as well as cancer with distant metastasis and para-aortic modal metastasis. In this IPD meta-analysis, all patients with stage III or stage IV gastric cancer were considered eligible, except for those with distant metastasis or paraaortic nodal metastasis. In addition, patients were excluded from the analysis if the data for survival duration or status were not available or if a noncurative resection had been performed. The primary end point was OS, which was defined as the time between the operation date and either the date of death from any cause or the last follow-up date. For surviving patients, the last follow-up date was treated as the censoring date.

\section{Statistical methods}

IPD meta-analysis is a type of analysis method for systematic reviews. We analyzed the IPD directly rather than extracting tabulated data from publications. The data were obtained directly from the investigators. Survival curves were estimated using the Kaplan-Meier method and were compared using a stratified log-rank test. A stratified proportional hazards model was used to estimate the hazard ratio (HR) and $95 \%$ confidence interval (CI) for the effects of OK-432. The trial was the stratification factor. Cochran's $Q$ and $I^{2}$ statistics for inconsistency [24] were used to assess statistical heterogeneity across the trials, and publication bias was assessed using a funnel plot. The effects of OK-432 in the different subgroups were investigated using stratified analysis with an interaction term. Forest plots were drawn to display the HRs by trial or by subgroup. A multivariate Cox model was fitted to the data to estimate the HR for OK-432, with adjustment for potential confounding factors found to be associated with OS $(p<0.10)$ in the univariate analyses. All $p$ values were two-sided. All analyses were performed using SAS 9.4 (SAS Institute, Cary, NC, USA) and Comprehensive Meta-Analysis (Biostat, Englewood, NJ, USA).

\section{Results}

We identified 67 abstracts from MEDLINE and nine abstracts from other sources. Abstracts from 16 studies were eligible (Fig. 1). The authors of 14 of the 16 eligible trials [9] were asked for and provided IPD ("Appendix"). We analyzed 1522 patients with stage III or stage IV gastric cancer. There were 796 patients in the OK-432 group and 726 patients in the control group. The numbers of patients in the OK-432 and control groups were well balanced in all trials. One trial was conducted in Korea, and the other 13 trials were conducted in Japan. Central randomization was

Table 1 Study design of randomized clinical trials using OK-432 in patients with stage III or stage IV gastric cancer

\begin{tabular}{|c|c|c|c|c|c|}
\hline & \multirow[t]{2}{*}{ Trial } & \multirow[t]{2}{*}{ Randomization } & \multirow[t]{2}{*}{ Chemotherapy } & \multicolumn{2}{|c|}{ No. of patients } \\
\hline & & & & OK-432 & Control \\
\hline $1 \mathrm{~A}$ & Aichi Cancer Center Hospital & Envelope & $\mathrm{MMC}$ (iv) $+5-\mathrm{FU}$ & 14 & 19 \\
\hline $3 \mathrm{~A}$ & Kyoto Research Group for Digestive Organ Surgery & Envelope & MMC (iv) + 5-FU or FT-207 or HCFU & 75 & 56 \\
\hline $4 \mathrm{~A}$ & JFMC (project \#4) & Central & MMC (iv) + UFT & 23 & 23 \\
\hline $5 \mathrm{~A}$ & JFMC (project \#5) & Central & MMC (iv) + FT-207 & 108 & 88 \\
\hline $6 \mathrm{~A}$ & JFMC (project \#17) & Central & MMC (iv) + UFT & 188 & 171 \\
\hline $7 \mathrm{~A}$ & CSGIGC & Envelope & $\mathrm{MMC}$ (iv) $+5-\mathrm{FU}$ & 42 & 45 \\
\hline $8 \mathrm{~A}$ & Osaka OK-432 Study Group & Central & MMC (iv) + UFT & 29 & 29 \\
\hline $9 \mathrm{~A}$ & Kyushu University & Envelope & MMC (iv) + UFT & 12 & 12 \\
\hline $11 \mathrm{~A}$ & Kyung Hee University & Envelope & MMC (iv) + 5-FU (iv) + ADM (iv) & 29 & 33 \\
\hline $12 \mathrm{~A}$ & Hokkaido University Group (study \#2) & Central & $\mathrm{MMC}$ (iv) + HCFU & 58 & 55 \\
\hline $13 \mathrm{~A}$ & Hokkaido University Group (study \#3) & Central & $5^{\prime}$-DFUR & 49 & 37 \\
\hline $14 \mathrm{~A}$ & Ojiya General Hospital & Envelope & MMC (iv) $+5-F U$ or UFT & 5 & 3 \\
\hline $15 \mathrm{~A}$ & Teikyo University & Envelope & MMC (iv) + UFT & 7 & 3 \\
\hline $16 \mathrm{~A}$ & SPOG & Central & $\mathrm{MMC}(\mathrm{iv})+5-\mathrm{FU}$ & 157 & 152 \\
\hline Total & & & & 796 & 726 \\
\hline
\end{tabular}

$A D M$ adriamycin, CSGIGC Clinical Study Group of Immunotherapy for Gastric Cancer, $5^{\prime}-D F U R$ doxifluridine, FT-207 tegaful, 5-FU 5-fluorouracil, $H C F U$ carmoful, iv intravenously, JFMC Japanese Foundation for Multidisciplinary Treatment of Cancer, $M M C$ mitomycin C, SPOG Study of Postoperative Adjuvant Immunochemotherapy using OK-432 for Gastric Cancer, UFT tegaful and uracil 
Table 2 Patient characteristics

\begin{tabular}{|c|c|c|c|}
\hline & OK-432 & Control & $p$ \\
\hline \multicolumn{4}{|l|}{ Gender } \\
\hline Male & $539(67.7 \%)$ & $475(65.4 \%)$ & \multirow[t]{2}{*}{0.345} \\
\hline Female & $257(32.3 \%)$ & $251(34.6 \%)$ & \\
\hline Median age (years) ${ }^{\mathrm{a}}$ & $60(52-67)$ & $61(51-67)$ & 0.535 \\
\hline \multicolumn{4}{|l|}{ pT } \\
\hline $\mathrm{T} 2$ & $229(28.8 \%)$ & $203(28 \%)$ & \multirow[t]{3}{*}{0.83} \\
\hline $\mathrm{T} 3$ & $484(60.8 \%)$ & $441(60.7 \%)$ & \\
\hline $\mathrm{T} 4$ & $83(10.4 \%)$ & $82(11.3 \%)$ & \\
\hline \multicolumn{4}{|l|}{$\mathrm{pN}$} \\
\hline N0 & $15(1.9 \%)$ & $17(2.3 \%)$ & \multirow[t]{3}{*}{0.322} \\
\hline N1 & $300(37.7 \%)$ & $248(34.2 \%)$ & \\
\hline $\mathrm{N} 2$ & $481(60.4 \%)$ & $461(63.5 \%)$ & \\
\hline \multicolumn{4}{|l|}{ M } \\
\hline M0 & 796 & 726 & \\
\hline \multicolumn{4}{|l|}{ Stage } \\
\hline III & $766(96.2 \%)$ & $685(94.3 \%)$ & \multirow[t]{2}{*}{0.083} \\
\hline IV & $30(3.8 \%)$ & $41(5.7 \%)$ & \\
\hline \multicolumn{4}{|l|}{ Splenectomy } \\
\hline No & $536(67.3 \%)$ & $458(63.1 \%)$ & \multirow[t]{2}{*}{0.082} \\
\hline Yes & $260(32.7 \%)$ & $268(36.9 \%)$ & \\
\hline \multicolumn{4}{|c|}{ Delayed-type hypersensitivity } \\
\hline Negative & $170(42.6 \%)$ & $148(39.9 \%)$ & \multirow[t]{3}{*}{0.445} \\
\hline Positive & $229(57.4 \%)$ & $223(60.1 \%)$ & \\
\hline Unknown & 397 & 355 & \\
\hline
\end{tabular}

a The interquartile range is given in parentheses.

performed in seven trials. Some studies included a very small number of patients (Table 1).

The patient characteristics are listed in Table 2. There were 539 male patients $(67.7 \%)$ in the OK-432 group and 475 male patients $(65.4 \%)$ in the control group. The median age was 60 years, and was balanced between the groups. The other patient characteristics were also balanced between both groups (Table 2). The median follow-up duration was 35.0 months (interquartile range 16.0-61.0 months). The median duration of OS was slightly different between the two groups (42.6 months in the OK-432 group and 32.3 months in the control group). During the follow-up period, 456 patients in the OK-432 group and 449 patients in the control group died (Fig. 2).

The number of events in each group and the HRs are listed in Fig. 3. Only one study (no. 9A) demonstrated a significant benefit of OK-432 therapy. One study (no. 14A) was extremely small and had no event in the OK-432 group; therefore, the standard error of the HR could not be calculated, and this study did not contribute to the estimation of the overall HR. There was a significant difference between the two groups, with an overall HR of 0.88

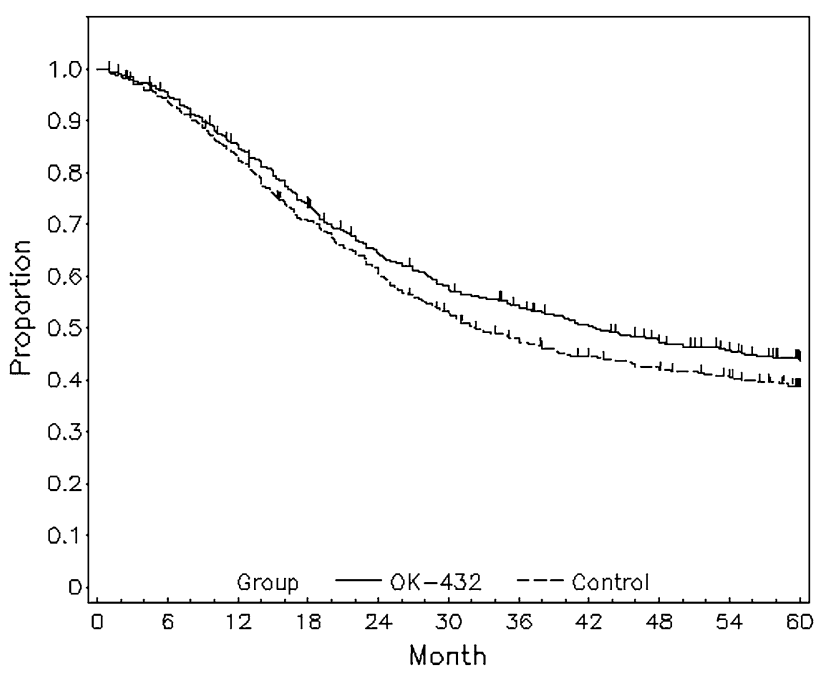

Fig. 2 Overall survival estimates after surgery truncated at 60 months. The survival rates were estimated using the KaplanMeier method

(95\% CI $0.77-1.00, p=0.050$ ). This corresponds to a $12 \%$ reduction in the risk with OK-432 treatment. No significant heterogeneity of the treatment effects was detected across the trials $\left(p=0.370, I^{2}=7.49 \%\right.$; Fig. 3$)$.

A funnel plot is shown in Fig. 4. Horizontal and vertical axes are point estimates of the log HR and its standard errors of individual studies. Extremely small studies tended to have smaller HRs.

The subgroup analyses according to the prespecified prognostic factors are shown in Fig. 5. The observed OK432 treatment effects were slightly greater in male patients than in female patients $(p=0.188)$. However, all factors showed no differences in the HRs between subgroups in interaction tests. The HR for OK-432 treatment based on centrally randomized trials $(4 \mathrm{~A}, 5 \mathrm{~A}, 6 \mathrm{~A}, 8 \mathrm{~A}, 12 \mathrm{~A}, 13 \mathrm{~A}$, and 16A) was not different from the overall estimate; this could hence be viewed as a sensitivity analysis, and indicated the robustness of the primary result. The covariate adjusted HR (0.91, $95 \%$ CI $0.80-1.04)$, estimated by the multivariate Cox model with adjustment for $\mathrm{pT}$, pN, stage, and splenectomy, was similar to the unadjusted value.

\section{Discussion}

Immunotherapy was once expected to become the ultimate treatment for various cancers, replacing the three major anticancer modalities of surgery, radiotherapy, and cytotoxic chemotherapy. However, most of the clinical trials evaluating the effects of immunopotentiators have failed to demonstrate a substantial benefit of treatment during the early phases of development. Beginning in the twenty-first century, molecularly targeted agents and monoclonal 


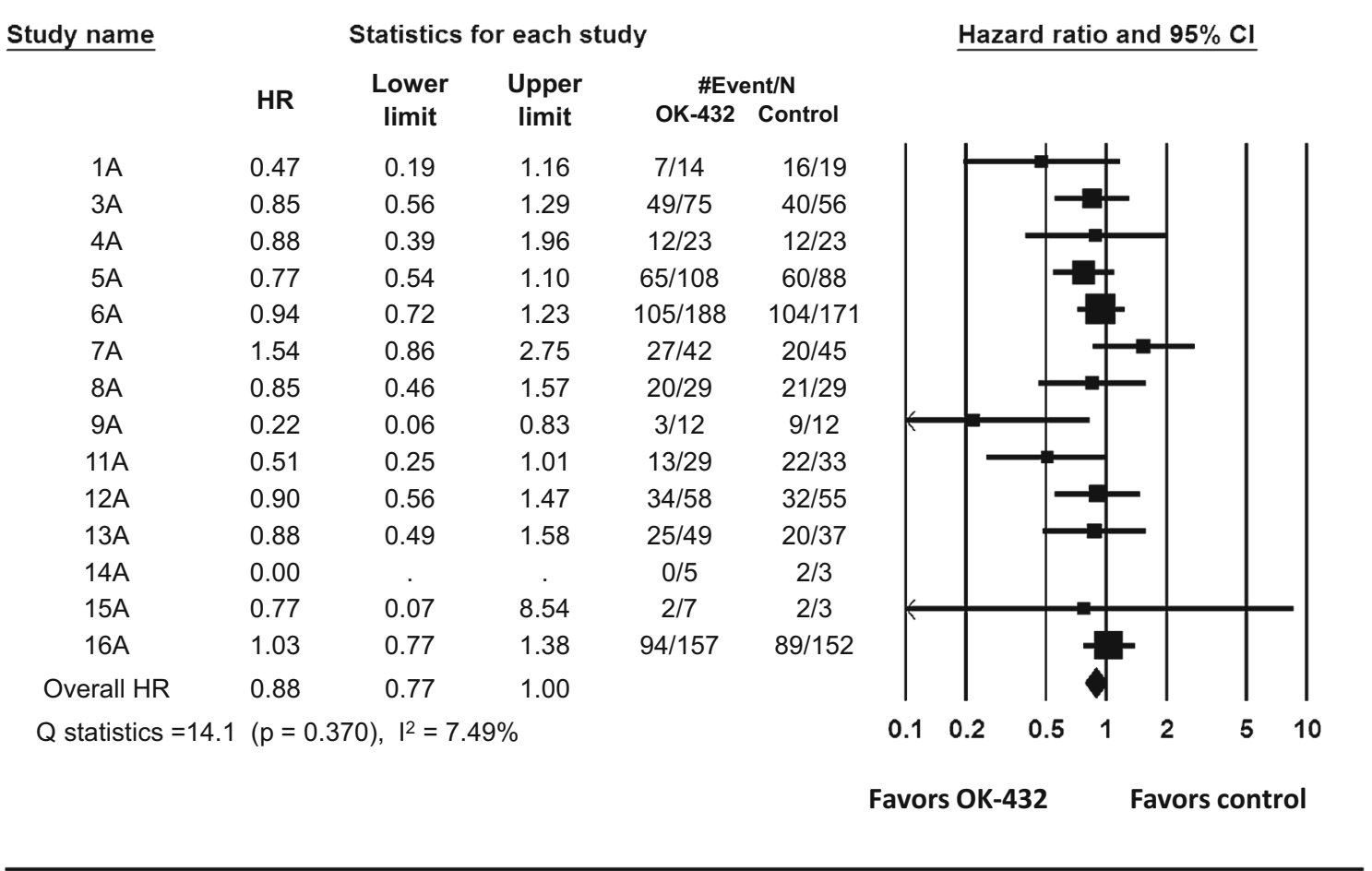

Fig. 3 Number of events and hazard ratios for the trials and the overall population. The $Q$ statistic and the $I^{2}$ statistic were used to evaluate heterogeneity between trials. Boxes and horizontal lines in

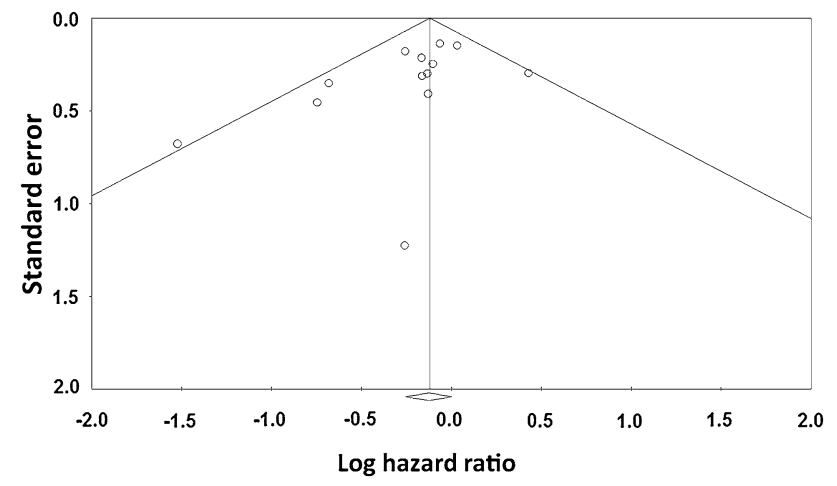

Fig. 4 Funnel plot depicting potential publication bias. The horizontal axis and the vertical axis reflect point estimates of the log hazard ratio and its standard error of individual studies, respectively

antibodies against specific cell or vascular endothelial growth factors, such as nivolumab and RG7446, were demonstrated to have clinical benefits in several cancer patients [25-30]. More recently, nonspecific immune activation systems have been shown to exert antitumor effects through direct intervention in the immune tolerance system. Given these new aspects of cancer immunotherapy, the importance of nonspecific immune activation by OK432 in relation to the suggested new synergistic mechanism involving immunopotentiators and tumor vaccines has the forest plot represent point estimates, which vary in size according to the weight in the analysis, and $95 \%$ confidence intervals, respectively. $C I$ confidence interval, $H R$ hazard ratio

been highlighted. In this regard, a precise review of the previous clinical trials using OK-432 was considered essential for further investigation.

In this study, we performed the evidence synthesis of adjuvant immunochemotherapy with OK-432 treatment for stage III or stage IV gastric cancer without metastasis using IPD instead of tabulated data from previous analyses. The results revealed a $12 \%$ risk reduction and a prolongation of survival (median 10 months), and suggested that the prognosis could be improved with this immunochemotherapy. Subgroup analysis of only the central randomized trials provided a similar result (HR 0.92, $95 \%$ CI 0.79-1.06), demonstrating the robustness of the primary results regardless of the quality of the clinical trials.

In this research, we could have focused on the appropriate population, comprising stage III gastric cancer patients and curatively resected stage IV gastric cancer patients who required more powerful adjuvant treatment than chemotherapy alone. Generally, the reliability of the evidence from a subgroup analysis is not adequate in a single randomized controlled trial because of the problems of multiplicity and underpower due to the limited sample size. In this study with the large amount of IPD from 14 trials, we could investigate the precise effects of immunochemotherapy with OK-432. Furthermore, we could have tried to eliminate confounding and bias using a 


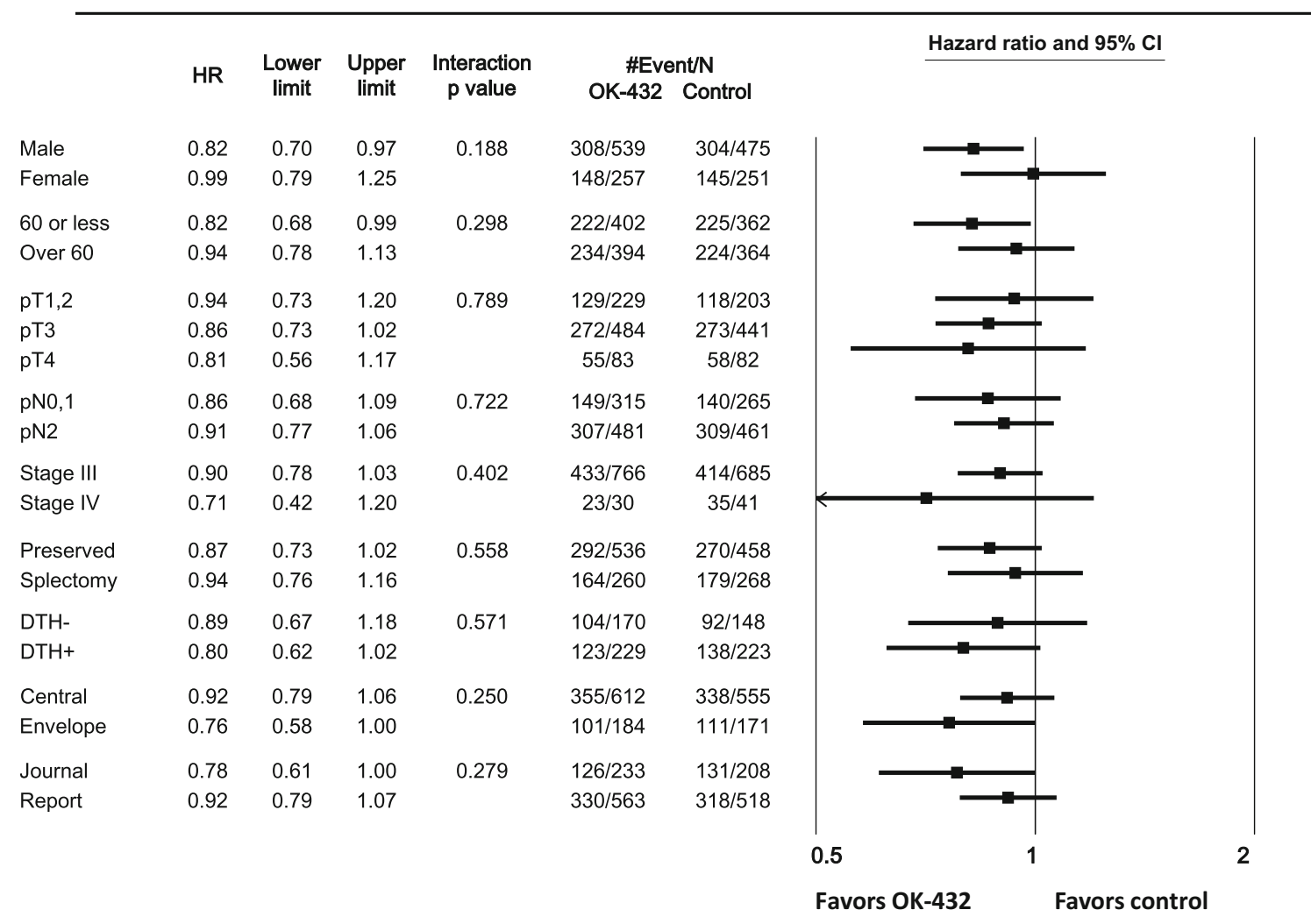

Fig. 5 Number of events and hazard ratios for the subgroups according to prognostic factors. The interaction $p$ value represents heterogeneity between the subgroups. Boxes and horizontal lines in the forest plot represent point estimates, which vary in size according

multivariate analysis that adjusted for prognostic factors, and confirmed the robustness of the result.

In the subgroup analysis, the spleen-preserved group showed a higher HR than the splenectomy group, although the difference was not statistically significant. Some molecular findings support the hypothesis that the presence of the spleen enhances the antitumor immune response [31, 32]. For instance, Okinaga et al. [33] conducted a randomized trial to investigate the efficacy of spleen preservation and immunochemotherapy with OK-432 for gastric cancer patients. They reported that spleen preservation might be beneficial for the immunological function of patients with less-advanced gastric cancer. However, the differences in survival rates were not statistically significant, and the clinical benefit remains unclear.

Other immunochemotherapies have also been investigated for the treatment of gastric cancer. For example, Popiela et al. [34] evaluated the effects of adjuvant immunochemotherapy with the use of bacillus CalmetteGuérin, and 5-fluorouracil, adriamycin, and mitomycin C, and reported a $24 \%$ risk reduction in patients with stage III or stage IV gastric cancer after curative resection. Oba to the weight in the analysis, and $95 \%$ confidence intervals, respectively. $C I$ confidence interval, DTH delayed-type hypersensitivity, $H R$ hazard ratio

et al. [35] performed a meta-analysis of immunochemotherapy with polysaccharide $\mathrm{K}$ for gastric cancer after curative resection, and reported a $12 \%$ risk reduction. Fujimoto et al. [36] reported the effects of postoperative adjuvant immunochemotherapy with the polysaccharide sizofiran on the same population, and concluded that curative resection of gastric cancer resulted in a better prognosis when sizofiran was prescribed along with the antitumor drug.

In terms of the treatment of other cancers with OK-432, Sakamoto et al. [37] reported a significant benefit of OK432 treatment for patients with resectable non-small-cell lung cancer. Watanabe et al. [38] conducted a randomized controlled tria involving patients with stage II-IV colorectal cancer. The results showed no significant differences in 5-year survival, but the combinations of OK-432 and an oral pyrimidine (1-hexylcarbamoyl-5-fluorouracil) or tegaful and uracil were well tolerated [38]. Most of the studies involving immunochemotherapy reported marginal results. This means that a larger clinical trial and simultaneous measurement of immunological parameters may be necessary to elucidate the underlying mechanism. 
A limitation of this research was that the trials with fewer deaths tended to have smaller HRs. However, this is not likely due to publication bias because we performed a comprehensive literature search and obtained data from trials those were not published, and our research focused only on patients with stage III or stage IV gastric cancer among all the patients from the eligible trials. Further, data from two trials were not obtained. One of these trials reported the efficacy of OK-432 $(N=236$ stage III gastric cancer patients, estimated HR 0.65), whereas the other did not $(N=211$ stage II and stage III gastric cancer patients, estimated HR 1.12). If these data were included in the analysis, the overall HR would have become smaller. Finally, because the trials included in this research were completed over a decade ago, the surgical methods and treatments could have changed since then. However, we confirmed that no new clinical trial has been implemented, and believe that a detailed analysis of the old clinical trial data is of substantial importance, given that immunotherapy for cancer has been highlighted recently and OK-432 is one of the key substances as an adjuvant for vaccine therapy. In addition, the collection and registration of all the IPD will facilitate approaches for analyzing tissue specimens from patients enrolled in previous clinical trials that might be exploited for future research in the new era of immunotherapy.

In conclusion, this IPD meta-analysis suggests that immunochemotherapy with OK-432 could have a borderline significant effect for patients with stage III or stage IV gastric cancer after curative resection. Further investigations related to the new findings of immune tolerance systems and nonspecific immunopotentiators are warranted.

Acknowledgment This work was supported in part by the Epidemiological and Clinical Research Information Network, a nonprofit organization.

\section{Appendix}

The IPD were obtained from the following trials (the study number refers to Sakamoto et al. [9]). Data were provided by: Yoshitaka Yamamura (1A), Yoshinori Nio (3A), the Japanese Foundation for Multidisciplinary Treatment of Cancer and Hiroaki Nakazato (4A, 5A and 6A), Kenji Ogawa (7A), Tetsuo Taguchi (8A), Yoshiyuki Maehara (9A), Kim Si-Young (11A), Yuji Sato (12A and 13A), Tadahiro Yokomori (14A), Kota Okinaga (15A), and Chugai Pharmaceutical and Kunzo Orita (16A):

1A Yamamura Y, Nishimura M, Sakamoto J, Yasui K, Morimoto T, Kato T, et al. A randomized controlled trial of surgical adjuvant therapy with mitomycin $\mathrm{C}$, 5-fluorouracil, and OK-432 for patients suffering from gastric cancer (in Japanese). Gan To Kagaku Ryoho. 1986;13:2134-40.

3A Kyoto Research Group for Digestive Organ Surgery. A comprehensive multi-institutional study on postoperative adjuvant immunotherapy with oral streptococcal preparation OK-432 for patients after gastric cancer surgery. Ann Surg. 1992;216:44-54.

4A Japanese Foundation for Multidisciplinary Treatment of Cancer. Report of project \#4 (in Japanese). Tokyo, Japan, 1995.

5A Japanese Foundation for Multidisciplinary Treatment of Cancer. Report of project \#5 (in Japanese). Tokyo, Japan, 1998.

6A Japanese Foundation for Multidisciplinary Treatment of Cancer. Report of project \#17 (in Japanese). Tokyo, Japan, 1999.

7A Ogawa K, Yagawa H, Katsube T, Kajiwara T, Ozawa T, Hanaoka T, et al. Studies on the effects of postoperative adjuvant immunotherapy with OK-432 on advanced gastric cancer. Proceedings of the XVI International Cancer Congress. 1994;16:1765-9.

8A Taguchi T. Data of industry. Osaka, Japan, 1993.

9A Maehara Y, Okuyama T, Kakeji Y, Baba H, Furusawa M, Sugimachi K, et al. Postoperative immunochemotherapy including streptococcal lysate OK-432 is effective for patients with gastric cancer and serosal invasion. Am J Surg. 1994;168:36-40.

11A Kim SY, Park HC, Yoon C, Yoon HJ, Choi YM, Cho KS. OK-432 and 5-fluorouracil, doxorubicin, and mitomycin C (FAM-P) versus FAM chemotherapy in patients with curatively resected gastric carcinoma. Cancer. 1998;83:2054-9.

12A Uchino J. Data of industry of study \#2. Hokkaido, Japan, 1996.

13A Uchino J. Data of industry of study \#3. Hokkaido, Japan, 1998.

14A Yokomori T, Iesato H, Kobayashi I, Watanuki K, Tokumine M, Muraoka M, et al. Adjuvant immunochemotherapy for gastric cancer with OK-432 (in Japanese). Biotherapy. 1999;13:201-5.

15A Okinaga $\mathrm{K}$, Iinuma $\mathrm{H}$, Fukushima $\mathrm{R}$, Yokohata $\mathrm{T}$, Kitamura Y, Shiraishi M. Effect of immunotherapy and spleen preservation on immunologic function in patients with gastric cancer (in Japanese). Geka Chiryo. 1999;78:605-11.

16A Orita K, Ohashi Y. Data of industry (study of postoperative adjuvant immunochemotherapy using OK-432 for gastric cancer). Tokyo, Japan, 2002. 


\section{References}

1. Okamoto H, Shoin S, Koshimura S, Shimizu R. Studies on the anticancer and streptolysin-forming abilities of hemolytic streptococci. Jpn J Microbiol. 1967;11:323-6.

2. Maehara Y, Okuyama T, Kakeji Y, Baba H, Furusawa M, Sugimachi K. Postoperative immunochemotherapy including streptococcal lysate OK-432 is effective for patients with gastric cancer and serosal invasion. Am J Surg. 1994;168:36-40.

3. Watanabe Y, Iwa T. Clinical value of immunotherapy with the streptococcal preparation OK-432 in non-small cell lung cancer. J Biol Response Mod. 1987;6:169-80.

4. Toge T, Yamada H, Aratani K, Kameda A, Kuroi K, Hisamatsu $\mathrm{K}$, et al. Effects of intraperitoneal administration of OK-432 for patients with advanced cancer. Jpn J Surg. 1985;15:260-5.

5. Uchida A, Micksche M. Intrapleural administration of OK432 in cancer patients: activation of NK cells and reduction of suppressor cells. Int J Cancer. 1983;31:1-5.

6. Sugiyama M, Yamane H, Cho JS, Okada H, Sugita M, Nakai Y. Enhanced production of gamma-interferon by therapy with parenteral OK-432 and alpha-interferon in patients with head and neck cancer. Arch Otorhinolaryngol. 1986;243:281-7.

7. Makimoto K, Ohmura M, Hoshino T. Immunologic parameters in patients with thyroid cancer. Arch Otorhinolaryngol. 1986;243: 91-5.

8. Ogita S, Tsuto T, Nakamura K, Deguchi E, Iwai N. OK-432 therapy in 64 patients with lymphangioma. J Pediatr Surg. 1994;29:784-5.

9. Sakamoto J, Teramukai S, Nakazato H, Sato Y, Uchino J, Taguchi T, et al. Efficacy of adjuvant immunochemotherapy with OK-432 for patients with curatively resected gastric cancer: a meta-analysis of centrally randomized controlled clinical trials. J Immunother. 2002;25:405-12.

10. Nakahara S, Tsunoda T, Baba T, Asabe S, Tahara H. Dendritic cells stimulated with a bacterial product, OK-432, efficiently induce cytotoxic $\mathrm{T}$ lymphocytes specific to tumor rejection peptide. Cancer Res. 2003;63:4112-8.

11. Ryoma Y, Moriya Y, Okamoto M, Kanaya I, Saito M, Sato M. Biological effect of OK-432 (Picibanil) and possible application to dendritic cell therapy. Anticancer Res. 2004;24:3295-302.

12. Vacchelli E, Eggermont A, Sautès-Fridman C, Galon J, Zitvogel L, Kroemer G, et al. Trial watch: toll-like receptor agonists for cancer therapy. Oncoimmunology. 2013;2:e25238.

13. Wada H, Isobe M, Kakimi K, Mizote Y, Eikawa S, Sato E, et al. Vaccination with NY-ESO-1 overlapping peptides mixed with Picibanil OK-432 and montanide ISA-51 in patients with cancers expressing the NY-ESO-1 antigen. J Immunother. 2014;37:84-92.

14. Endo H, Saito T, Kenjo A, Hoshino M, Terashima M, Sato T, et al. Phase I trial of preoperative intratumoral injection of immature dendritic cells and OK-432 for resectable pancreatic cancer patients. J Hepatobiliary Pancreat Sci. 2012;19:465-75.

15. Eikawa S, Kakimi K, Isobe M, Kuzushima K, Luescher I, Ohue Y. Induction of CD8 $\mathrm{T}$ cell responses restricted to multiple HLA class I alleles in a cancer patient by immunization with a 20-mer NY-ESO-1f (NY-ESO-1 91-110) peptide. Int J Cancer. 2013;132: $345-54$.

16. Koido S, Homma S, Okamoto M, Takakura K, Mori M, Yoshizaki S, et al. Treatment with chemotherapy and dendritic cells pulsed with multiple Wilms' Tumor 1 (WT1)-specific MHC class I/II-restricted epitopes for pancreatic cancer. Clin Cancer Res. 2014;20:4228-39.

17. Kobayashi M, Chiba A, Izawa H, Yanagida E, Okamoto M, Shimodaira S, et al. The feasibility and clinical effects of dendritic cell-based immunotherapy targeting synthesized peptides for recurrent ovarian cancer. J Ovarian Res. 2014;7:48.
18. Galluzzi L, Senovilla L, Vitale I, Michels J, Martins I, Kepp O, et al. Molecular mechanisms of cisplatin resistance. Oncogene. 2012;31:1869-83.

19. Chen WJ, Yuan SF, Yan QY, Xiong JP, Wang SM, Zheng WE, et al. Intrapleural chemo- and hyperthermotherapies for malignant pleural effusion: a randomized prospective study. Cancer Invest. 2012;30:126-30.

20. Hirayama $M$, Nishikawa $H$, Nagata $Y$, Tsuji $T$, Kato $T$, Kageyama $\mathrm{S}$, et al. Overcoming regulatory $\mathrm{T}$ cell suppression by a lyophilized preparation of streptococcus pyogenes. Eur J Immunol. 2013;43:989-1000.

21. Saito T, Tsuchiya T, Sato Y, Kenjo A, Kimura T, Anazawa T, et al. Effect of transarterial immunoembolization as preoperative treatment for hepatocellular carcinoma. Ann Cancer Res Ther. 2011;19:26-33.

22. Tano T, Okamoto M, Kan S, Bando T, Goda H, Nakashiro K, et al. Immunochemoradiotherapy for patients with oral squamous cell carcinoma: augmentation of OK-432-induced helper T cell 1 response by 5-FU and X-ray irradiation. Neoplasia. 2013;15:805-14.

23. Sakuramoto S, Sasako M, Yamaguchi T, Kinoshita T, Fujii M, Nashimoto A, et al. Adjuvant chemotherapy for gastric cancer with S-1, an oral fluoropyrimidine. N Engl J Med. 2007;357:1810-20.

24. Borenstein M, Hedges LV, Higgins JPT, Rothstein HR. Introduction to meta-analysis. Chichester: Wiley; 2009.

25. Van Cutsem E, Köhne CH, Láng I, Folprecht G, Nowacki MP, Cascinu S, et al. Cetuximab plus irinotecan, fluorouracil, and leucovorin as first-line treatment for metastatic colorectal cancer: updated analysis of overall survival according to tumor KRAS and BRAF mutation status. J Clin Oncol. 2011;29:2011-9.

26. de Gramont A, Van Cutsem E, Schmoll HJ, Tabernero J, Clarke S, Moore MJ, et al. Bevacizumab plus oxaliplatin-based chemotherapy as adjuvant treatment for colon cancer (AVANT): a phase 3 randomised controlled trial. Lancet Oncol. 2012;13:1225-33.

27. Yamazaki N, Tahara $\mathrm{H}$, Uhara $\mathrm{H}$, et al. Phase 2 study of nivolumab (anti-PD-1; ONO-4538/BMS-936558) in patients with advanced melanoma. Amsterdam: European Cancer Congress; 2013

28. Robert C, Antonio Ascierto P, Maio M, et al. A phase III, randomized, double-blind study of nivolumab (anti-PD-1; BMS936558; ONO-4538) versus dacarbazine in patients (pts) with previously untreated, unresectable, or metastatic melanoma (MEL). J Clin Oncol 2013;31(15 Supp):TPS9106.

29. Rizvi NA, Chow LQM, Dirix LY, Gettinger SN, Gordon MS, Kabbinavar FF, et al. Clinical trials of MPDL3280A (anti-PDL1) in patients (pts) with non-small cell lung cancer (NSCLC). J Clin Oncol. 2014;32:(5 Suppl):TPS8123.

30. Bocchia M, Bronte V, Colombo MP, De Vincentiis A, Di Nicola M, Forni G, et al. Antitumor vaccination: where we stand. Haematologica. 2000;85:1172-206.

31. Wakasugi T, Takeda T, Monden T, Katsumoto Y, Sakita I, Nagaoka $\mathrm{H}$, et al. Augmentation of splenic antitumor immunity by local immunotherapy in gastric cancer patients. Biotherapy. 1997;10:99-106.

32. Fujie T, Tanaka F, Tahara K, Li J, Tanaka S, Mori M, et al. Generation of specific antitumor reactivity by the stimulation of spleen cells from gastric cancer patients with MAGE-3 synthetic peptide. Cancer Immunol Immunother. 1999;48:189-94.

33. Okinaga $K$, Iinuma $H$, Kitamura $Y$, Yokohata $T$, Inaba $T$, Fukushima R. Effect of immunotherapy and spleen preservation on immunological function in patients with gastric cancer. J Exp Clin Cancer Res. 2006;25:339-49.

34. Popiela T, Kulig J, Czupryna A, Szczepanik AM, Zembala M. Efficiency of adjuvant immunochemotherapy following curative resection in patients with locally advanced gastric cancer. Gastric Cancer. 2004;7:240-5. 
35. Oba K, Teramukai S, Kobayashi M, Matsui T, Kodera Y, Sakamoto J. Efficacy of adjuvant immunochemotherapy with polysaccharide $\mathrm{K}$ for patients with curative resections of gastric cancer. Cancer Immunol Immunother. 2007;56:905-11.

36. Fujimoto S, Furue H, Kimura T, Kondo T, Orita K, Taguchi T, et al. Clinical outcome of postoperative adjuvant immunochemotherapy with sizofiran for patients with resectable gastric cancer: a randomised controlled study. Eur J Cancer. 1991;27:1114-8.

37. Sakamoto J, Teramukai S, Watanabe Y, Hayata Y, Okayasu T, Nakazato H, et al. Meta-analysis of adjuvant immunochemotherapy using OK-432 in patients with resected non-small-cell lung cancer. J Immunother. 2001;24:250-6.

38. Watanabe M, Nishida O, Kunii Y, Kodaira S, Takahashi T, Tominaga $\mathrm{T}$, et al. Randomized controlled trial of the efficacy of adjuvant immunochemotherapy and adjuvant chemotherapy for colorectal cancer, using different combinations of the intracutaneous streptococcal preparation OK-432 and the oral pyrimidines 1-hexylcarbamoyl-5-fluorouracil and uracil/tegafur. Int $\mathrm{J}$ Clin Oncol. 2004;9:98-106. 